\title{
Two Dimensional Grayscale Images of the Aspiny Neurons from the Human Neostriatum: Monofractal and Gray Level Co-occurrence Matrix Analysis
}

\author{
Velicko Vranes ${ }^{1, ~ *, ~ B o j a n a ~ K r s t o n o s ̌ i c ́ ~}{ }^{2}$, Nebojša Tomislav Miloševićc ${ }^{1,3}$ \\ ${ }^{1}$ Department of Basic and Environmental Science, Instituto Tecnológico de Santo Domingo (INTEC), Santo Domingo, Dominican Republic \\ ${ }^{2}$ Department of Anatomy, Faculty of Medicine, University of Novi Sad, Novi Sad, Serbia \\ ${ }^{3}$ Department of Biophysics, Faculty of Medicine, University of Belgrade, Belgrade, Serbia
}

Email Addresses:

velicko.vranes@intec.edu.do (V. Vranes), bkrstonosic@gmail.com (B. Krstonošić), nebojsa.milosevic@med.bg.ac.rs (N. T. Milošević)

${ }^{*}$ Corresponding author

\section{To cite this article:}

Velicko Vranes, Bojana Krstonošić, Nebojša Tomislav Milošević. Two Dimensional Grayscale Images of the Aspiny Neurons from the Human Neostriatum: Monofractal and Gray Level Co-occurrence Matrix Analysis. European Journal of Biophysics.

Vol. 7, No. 1, 2019, pp. 15-22. doi: 10.11648/j.ejb.20190701.13

Received: June 19, 2019; Accepted: July 23, 2019; Published: August 12, 2019

\begin{abstract}
The striatum (neostriatum) is one of the principal constituents of the basal nuclei. It is a complex structure which consists of a dorsal and the ventral components. According to the spine distribution and their density, neurons of the human striatum can be classified into two main types: spiny and aspiny cells. Further classification recognizes two groups of spiny, and three groups of aspiny neurons. The goal of this study was to analyze different morphometric properties of the digital images of the group IV and group V aspiny neurons, from the dorsal striatum of both cerebral hemispheres. In this study, a total of 175 two-dimensional images of aspiny neurons were analyzed. Image reconstruction and measurement was performed with the specialized, public software Image J. Four parameters of standard fractal analysis were quantified from these binary images. In addition, five textural parameters were obtained by analyzing grayscale images of the entire neuron. Results of both analyses show that six of nine parameters differed between the group IV and group V aspiny neurons. Moreover, in both groups of neurons, one parameter of the fractal and three of the texture analyses differed between the putamen and the caudate nucleus neurons. Thus, this study corroborates previous classification of aspiny neurons. Although they belong to the same aspiny group, different type of cells can qualify nerve signals in their own way. Therefore, this study supports the hypothesis that neuronal morphology differences can reflect their functional diversity and their role in communication.
\end{abstract}

Keywords: GLCM Analysis, Human Adult, 2D Image, Monofractal Analysis, Morphology, Neostriatum

\section{Introduction}

The striatum (neostriatum) is one of the main components of the basal nuclei, which has a dominant role in controlling motor activity, but also participates in the control of various behavioral outputs [1]. Despite its complex structure, the dorsal and the ventral striatum are generally recognized [2]. The dorsal component consists of the putamen and the caudate nucleus, while the ventral one includes the nucleus accumbens and the olfactory tubercle [3].

The neostriatum is characterized by exceptional heterogeneity, both in functional and morphological terms. For instance, the ventral striatum plays a role in reward and reinforcement system, while the dorsolateral striatum is related to the movement control and belongs to the sensorimotor territory [4]. The most numerous type of nerve cells in the neostriatum are spiny projection neurons, while others are interneurons, or local circuit cells [5]. Their axons do not leave the neostriatum, mainly establishing connections with spiny projection neurons. Its activity is primarily defined by the stimulus brought from the cortex and thalamus [6].

Qualitative analysis of striatal nerve cells implies a detailed description of their structure. During the last century, such studies have characterized the morphology of nerve cells by describing the different properties of neurons [6-8]. 
Five types of neurons in the human neostriatum have been identified [9, 10]. In our previous studies, striatal neurons were described through qualitative and quantitative analysis $[11,12]$. According to the presence of spines on cell bodies and along dendrites, striatal neurons were primarily classified into two types: spiny and aspiny cells. Furthermore, qualitative classification was confirmed through quantitative analysis in which eight computational parameters were used to determine five aspects of the neuronal images [11].

The main objective of this study was to improve previous results [11] and to describe contrasting morphological features of different neuronal types in greater detail. To achieve this goal, four fractal and five textural parameters were used. Although two fractal parameters have been used previously $[11,12]$, we utilized a novel and improved method of calculation [13]. Moreover, grayscale imaging was used to analyze five neuronal properties for the first time. Another objective of this study was measuring morphological differences in group IV and group $\mathrm{V}$ aspiny neurons between different parts of the neostriatum, specifically the caudate nucleus and putamen. To this end, obtained data was discussed regarding the functional diversity of the neostriatal regions.

\section{Materials and Methods}

\subsection{Experimental Material and Image Acquisition}

Postmortem brain material was collected from thirty adult human individuals during medico-legal autopsies in the Centre for Forensic Medicine, Toxicology and Molecular Genetics at the Clinical Centre of Vojvodina (Serbia), from 2008 to 2010. There was no evidence of neurological diseases and the study was performed in accordance with the ethical standards defined by the 1964 Declaration of Helsinki. The protocol was approved by the Ethical Committee of the University of Novi Sad (Faculty of Medicine in Novi Sad, Serbia), as well as with the written informed consent of the next of kin of the deceased. Both human cerebral hemispheres were dissected within $24 \mathrm{~h}$ postmortem. Tissue blocks, $15 \times 15 \mathrm{~mm}$ in size, including the portions of adjoing structures, were processed according to the Golgi-Kopsch-Bubenaite method [12]. The complete procedure of histological preparation of the sample was described in a prior paper [11].

Table 1. Illustration of material used in the study. Number of human brains, the age arranged in six intervals and causes of death in each interval. Data were collected from [12], with permission.

\begin{tabular}{lll}
\hline $\begin{array}{l}\text { Age } \\
\text { (year) }\end{array}$ & $\begin{array}{l}\text { Number } \\
\text { of brains }\end{array}$ & Cause of death (COD) \\
\hline $31-40$ & 2 & $\begin{array}{l}\text { Aneurysma aortae thoracicalis ruptum (1) Vulnus } \\
\text { punctum colli (1) } \\
\text { Infarctus myocardii acutus (6) Aneurysma aortae } \\
\text { thoracicalis ruptum (1) } \\
\text { Ruptura cordis sine haemopericardio post } \\
\text { infarctum cordis acutum (1) } \\
\text { Perniones (1) }\end{array}$ \\
$51-50$ & 9 & $\begin{array}{l}\text { Fractura multiplex, non specificata (3) } \\
\text { Aneurysma aortae et dissection aortae (2) }\end{array}$ \\
\hline
\end{tabular}

\begin{tabular}{lll}
\hline $\begin{array}{l}\text { Age } \\
\text { (year) }\end{array}$ & $\begin{array}{l}\text { Number } \\
\text { of brains }\end{array}$ & Cause of death (COD) \\
\hline & & $\begin{array}{l}\text { Neoplasma malignum (1) } \\
\text { Infarctus myocardii recidivus acutus, partis } \\
\text { non specificata (5) } \\
\text { Neoplasma malignum (2) }\end{array}$ \\
$61-70$ & 7 & $\begin{array}{l}\text { Submersio (1) } \\
\text { Neoplasma malignum (1) } \\
\text { Submersio (1) } \\
\text { Suspensio (1) }\end{array}$ \\
\hline
\end{tabular}

After impregnation, the serial coronal sections were cut and mounted on glass slides. Each section was analyzed using the light microscope "Leica DMLS" (Leica Microsystems, Wetzlar, Germany), at the ocular and eyepiece magnification of $400 \mathrm{x}(10 \mathrm{x} 40)$. All tissue samples were recorded and transformed into digital images using the digital camera "Leica DC 100", with the software package "Digital Camera Systems" (Leica Microsystems, Heerbrugg, Switzerland). Bearing in mind dendritic arborization and dendritic length, each neuron from the sample was recorded in 4-20 focal planes. The images of planes were loaded into freely available Image J software (https://imagej.nih.gov/ij/) and projected onto a stack along the axis perpendicular to the image plane [12]. In the next step, cells were selected and cropped according to the following criteria: (i) no overlapping with the neighboring cells; and (ii) clearly visible whole cell bodies, dendrites and their branches. The final image of the neuron was saved in two formats: grayscale and binary (Figures $1 \mathrm{~A}$ and $1 \mathrm{~B})$. A total of 175 two-dimensional images of aspiny neurons were obtained, 89 from putamen and 86 from caudate nucleus.

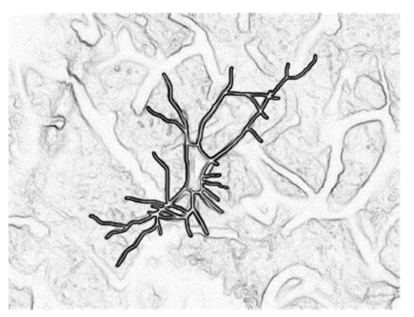

A

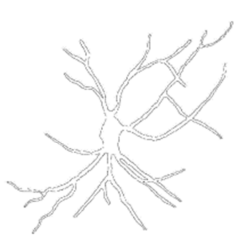

C

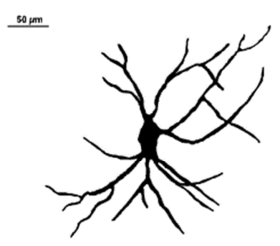

B
Figure 1. Representative digital image of group IV aspiny neuron from the human neostriatum. A) grayscale, B) binary, C) binary-outline and D) binary-skeleton image.

\subsection{Quantification of the Grayscale Image}

In general, grayscale images of neurons could be easily quantified when the technique of texture image analysis was used. This technique provides quantitative information of the 
distribution of gray color intensity within a group of pixels in an image. Precisely, it may well quantify: homogeneity of the tones in an image, linear connection of the tones, contrast of an image, boundary between the gray tones and complexity of the distribution of the tones [14].

In this study, one well-known texture technique - the Gray Level Co-occurrence Matrix (GLCM) was used. It is a twodimensional gray tone histogram for a selected pair of pixels, separated by a fixed spatial distance [15], in the form of a matrix. This technique calculates five parameters [16] that describe: i) texture uniformity of the image (angular second moment $S_{A S M}$ ), ii) local homogeneity of the image (inverse difference moment $S_{I D M}$ ), iii) spatial frequency of the gray tones in the image (contrast $S_{C O N}$ ), iv) distribution of the gray tones in the image (correlation $S_{C O R}$ ) and v) amount of information combined with complexity of the image (entropy $S_{E}$ ). Five textural features were calculated by the following formulas:

$$
S_{A S M}=\sum_{i} \sum_{j}\{p(i, j)\}^{2},
$$

$$
\begin{gathered}
S_{I D M}=\sum_{i} \sum_{j} \frac{1}{1+(i+j)^{2}} p(i, j), \\
S_{C O N}=\sum_{n=0}^{N_{g}-1} n^{2}\left\{\sum_{i=1}^{N_{g}} \sum_{j=1}^{N_{g}} p(i, j)\right\},|i-j|=n, \\
S_{C O R}=\sum_{i, j} \frac{\left(i-\mu_{x}\right)\left(j-\mu_{y}\right)}{\sqrt{\left(\sigma_{x} \sigma_{y}\right)}} p(i, j), \\
S_{E}=-\sum_{i} \sum_{j} p(i, j) \log (p(i, j)) .
\end{gathered}
$$

The term $N_{g}$ is the number of gray levels in the image. Their mathematical definitions and detailed explanations can be found in [17]. The meaning of all parameters (fractal and textural) used in this investigation are summarized in Table 2.

Table 2. Summary of nine computational parameters (monofractal and GLCM) of 2D binary and grayscale images of neurons from the human neostriatum. Each parameter is represented by a name, symbol and an explanation of what quantifies in $2 D$ image.

\begin{tabular}{llll}
\hline Parameters & Name & Symbol & Image Property \\
\hline \multirow{4}{*}{ Monofractal } & Binary box dimension & $D_{\text {bin }}$ & Space-filling in binary image \\
& Outline box dimension & $D_{\text {out }}$ & Shape in outline image \\
& Skeletonized box dimension & $D_{\text {skel }}$ & Irregularity and complexity in skeletonized image \\
& Lacunarity & $\Lambda$ & Heterogeneity of binary image in its simultaneous translation and rotation \\
& Angular second moment & $S_{A S M}$ & Texture uniformity in grayscale image \\
& Inverse difference moment & $S_{I D M}$ & Local homogeneity in grayscale image \\
& Contrast & $S_{C O N}$ & Spatial frequency of gray tones in grayscale image \\
& Correlation & $S_{C O R}$ & Distribution of the gray tones in grayscale image \\
& Entropy & $S_{E}$ & Amount of information with complexity of grayscale image
\end{tabular}

The GLCM technique was performed with the Texture Analyzer plugin [18] of Image $\mathrm{J}$ on the grayscale image (Figure 1A). The plugin was attuned as follows: the pixel distance was set to 1 and isotropic spatial orientation obtained by averaging the feature values at $0,90,180$ and $270^{\circ}$ angles.

\subsection{Quantification of the Binary Image}

Among various methods of computational analysis, the monofractal analysis appears to be the method which suitably computes natural objects, when they can be characterized with the unique value of the fractal dimension $[13,19]$. On the contrary, when objects possess an uneven distribution of complexity, with fractal dimension fluctuating from point to point within an object [20], then a multifractal analysis is appropriate. To perform a monofractal analysis, a digital image (RGB or grayscale) is converted into two characteristic presentations: binary and outline (previously known as "silhouette" image) [20]. The binary image represents compression of an initial image to two values (typically in 0 and 255). The outline image was created when one-pixel wide outline in a binary image was generated [13]. There is another type of image known as skeleton image of the object [21]. In most image software, command skeletonize works only with the binary 2D images, in the way that it removes pixels from the edges of an object until they are reduced to a single-pixel-wide shape. This type of image is particularly useful when neuronal images needs to be quantified. Examples of outline and skeleton neuronal images are illustrated in Figures $1 \mathrm{C}$ and $1 \mathrm{D}$.

The monofractal analysis was performed with the boxcounting method, i.e. the most frequently used method of fractal analysis [22]. This method is incorporated in Image $\mathrm{J}$, either with the standard command or with the appropriate plugin [23]. Using both, four parameters were obtained ( $D_{\text {bin }}, D_{\text {out }}, D_{\text {skel }}$ and $\Lambda$ ) for three types of each neuronal image (Figures 1B-1D). These parameters evaluate i) the space-filling ratio $\left(D_{\text {bin }}\right)$, ii) the shape $\left(D_{\text {out }}\right)$, iii) dendritic curvature and complexity of the dendritic tree $\left(D_{\text {skel }}\right)$, and iv) heterogeneity of the image in its simultaneous translation and rotation $(\Lambda)$. When the box counting method is applied, the $\Lambda$, is calculated by the number of pixels per all possible sizes of boxes. Generally, images with the larger or more numerous gaps have higher lacunarity, while those with the low values of lacunarity imply homogeneity based on similarly sized gaps and little rotational variance. 


\subsection{Statistical Analyses}

Distribution of the data was tested using three criteria (skewness, kurtosis and $p$ value of Kolmogorov-Smirnov test) which are presented in Table 3. Moreover, two monofractal and two GLCM parameters were plotted, and their possible correlation was investigated calculating Spearman correlation coefficient (Figure 2). The difference of monofractal and GLCM parameters between two types of neuronal images (and, also, between two striatal regions) was statistically analyzed by Mann-Whitney $(U)$ test. A value of $p<0.05$ was considered statistically significant. The SPSS software package v23 (IBM SPSS Statistics, Chicago, IL, USA, demo version) was employed for statistical analysis.

\section{Results}

There is no generally accepted procedure on the basis of which the normality of the distribution would be easily verified [24]. It is desirable that the researcher relies on more than one method for checking normality. Therefore, the parameter distribution properties on this sample were evaluated through three criteria: skewness $(a)$, kurtosis $(k)$ and $p$ value of Kolmogorov-Smirnov test (with Lilieffors correction). While the skewness is a measure of symmetry of distribution (more precisely, data set is symmetric if it looks the same to the left and right of the center point), the kurtosis is a measure of whether the data are heavy (or light)-tailed relative to a normal distribution. Finally, the results of the Kolmogorov-Smirnov test were used as criterion if data were normally distributed (Table 3 ).

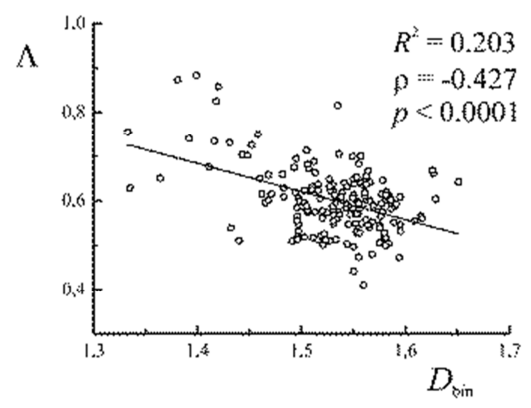

A

Table 3. Testing the distribution of 9 parameters $\left(D_{b i n}, D_{o u t}, D_{\text {skel }}, \Lambda, S_{A S M}\right.$, $S_{I D M}, S_{C O N}, S_{C O R}$ and $S_{E}$ ) on a sample of 175 aspiny neurons of the human stratum. For all parameters the skewness (a), kurtosis $(k)$ and $p$ value of the Kolmogorov-Smirnov test were calculated and displayed.

\begin{tabular}{llll}
\hline Parameter & $\boldsymbol{a}$ & $\boldsymbol{k}$ & $\boldsymbol{p}^{*}$ \\
\hline$D_{\text {bin }}$ & -1.082 & 1.828 & 0.001 \\
$D_{\text {out }}$ & $0.345^{* *}$ & 4.141 & 0.029 \\
$D_{\text {skel }}$ & $-0.367^{* *}$ & $0.393^{* * *}$ & 0.030 \\
$\Lambda$ & $0.989^{* *}$ & 2.319 & 0.003 \\
$S_{A S M}$ & $0.977^{* *}$ & $0.776^{* * *}$ & 0.000 \\
$S_{I D M}$ & $0.294^{* *}$ & -1.124 & 0.000 \\
$S_{C O N}$ & $0.547 * *$ & $-0.182^{* * *}$ & 0.022 \\
$S_{C O R}$ & 6.033 & 55.253 & 0.000 \\
$S_{E}$ & $-0.056^{* *}$ & -1.235 & 0.000 \\
\hline
\end{tabular}

*Lilliefors Significance Correction (the hypothesis that data come from a normally distributed population, when the hypothesis does not specify the expected value and variance of the distribution),

**Symmetric distribution $\mathrm{a} \in(-1,1)$,

*** Light tailed to the normal distribution $\mathrm{k} \in(-1,1)$,

${ }^{* * *}$ Normal distribution $p>0.05$

In previous publications it has been shown that some parameters of the image can describe similar [25] or complementary [17] properties. Therefore, we tested possible relationship between two parameters of monofractal $\left(D_{\text {bin }}\right.$ and $\left.\Lambda\right)$ and textural $\left(S_{I D M}\right.$ and $\left.S_{E}\right)$ analysis. The relationship and the statistical analysis of the correlation between these parameters were shown in Figure 2. As can be seen from the figure, there was a negative correlation in both cases. What is more important, both correlations were statistically significant. In addition, there was a poor link between the first two parameters $\left(D_{\text {bin }}\right.$ and $\left.\Lambda\right)$, while between the other two $\left(S_{I D M}\right.$ and $\left.S_{E}\right)$ the link was robust. Consequently, all conclusions regarding the statistical estimation of the $\Lambda$ and $S_{E}$ were analogous to the results of the analysis of the $D_{\text {bin }}$ and $S_{I D M}$, respectively.

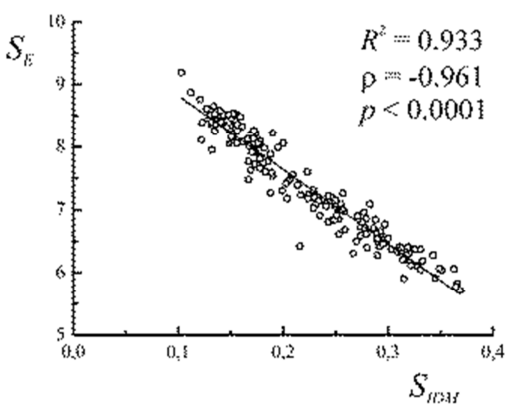

$\mathrm{B}$

Figure 2. Plot of $\Lambda$ versus $D_{\text {bin }}(A)$ and $S_{E}$ versus $S_{I D M}(B)$ for all sample of aspiny neurons from the human neostriatum. Data was fitted with straight line. The coefficient of determination $\left(R^{2}\right)$, Spearman coefficient of correlation $(\rho)$ and level of significance $(p)$ are inscribed in the plot.

All observed cells belong to two groups of human striatal interneurons: group IV and group V [11]. Considering the dendritic field area of these neurons, they can be referred to as large and small aspiny neurons, respectively. Therefore, we expanded previous computational analysis with monofractal and textural parameters. Our aim was to investigate whether these parameters can distinguish morphometric differences between two groups of aspiny neurons.
Table 3 shows significantly different parameters between groups. As can be seen, four parameters $\left(D_{\text {bin }}\right.$, $D_{\text {out }}, D_{\text {skel }}$ and $S_{C O R}$ ) have higher values in group IV. Contrary to the previous, two textural analysis parameters $\left(S_{C O N}\right.$ and $\left.S_{C O R}\right)$ have higher values in group V (Table 4). Summing up, six parameters were powerfully different: $D_{b i n}$ and $S_{A S M}$ were different at a significance level of 0.01 , while the remaining four parameters were different at a level less than 0.001 . 
Table 4. Statistically significant parameters of monofractal ( $D_{\text {bin }}$, $D_{\text {out }}$ and $\left.D_{\text {skel }}\right)$ and textural $\left(S_{A S M}, S_{C O N}\right.$ and $\left.S_{C O R}\right)$ analysis between two groups of aspiny neurons from the human neostriatum. Symbol $U$ is the value of Mann-Whitney test, while p denotes significance value.

\begin{tabular}{|c|c|c|c|c|c|c|}
\hline \multirow{2}{*}{ Parameter } & \multicolumn{2}{|c|}{ Group IV } & \multicolumn{2}{|l|}{ Group V } & \multirow{2}{*}{$U$} & \multirow[b]{2}{*}{$p$} \\
\hline & Median & Range & Median & Range & & \\
\hline$D_{\text {bin }}$ & 1.550 & 0.100 & 1.525 & 0.318 & 2787 & 0.002 \\
\hline$D_{\text {out }}$ & 1.297 & 0.375 & 1.245 & 0.238 & 1255.5 & $<0.001$ \\
\hline$D_{\text {skel }}$ & 1.190 & 0.170 & 1.135 & 0.323 & 1395.5 & $<0.001$ \\
\hline$S_{A S M}$ & 0.0020 & 0.0085 & 0.0030 & 0.0125 & 2866 & 0.004 \\
\hline$S_{\mathrm{CON}}$ & 644 & 1365 & 854 & 1416 & 2359.5 & $<0001$ \\
\hline$S_{C O R}$ & 0.00029 & 0.00071 & 0.00023 & 0.00226 & 2441.5 & $<0.001$ \\
\hline
\end{tabular}

Finally, the morphological differences of each group of the aspiny neurons between two parts of the neostriatum (putamen and caudate nucleus) were examined. The results of the statistical analysis of 9 parameters are shown in Table 4.

Table 5. Results of the Mann-Whitney (U) test of two groups (group IV and $V$ ) of aspiny neurons between two nuclei of the human neostriatum (putamen and caudate nucleus). The inequality $p<0.05$, determines a statistically significant difference between nuclei.

\begin{tabular}{lllll}
\hline \multirow{2}{*}{ Parameter } & Group IV & \multicolumn{3}{l}{ Group V } \\
\cline { 2 - 5 } & $\boldsymbol{U}$ & $\boldsymbol{p}$ & $\boldsymbol{U}$ & $\boldsymbol{p}$ \\
\hline$D_{\text {bin }}$ & 802 & 0.375 & 714 & 0.016 \\
$D_{\text {out }}$ & 622 & 0.013 & 969 & 0.734 \\
$D_{\text {skel }}$ & 641 & 0.021 & 980 & 0.802 \\
$\Lambda$ & 647 & 0.024 & 702 & 0.013 \\
$S_{A S M}$ & 61 & $<0.001$ & 253 & \\
$S_{I D M}$ & 43 & 0.592 & 569 & $<0.001$ \\
$S_{C O N}$ & 842 & 0.270 & 598 & 0.002 \\
$S_{C O R}$ & 778 & $<0.001$ & 9 & $<0.001$ \\
$S_{E}$ & 28 & & &
\end{tabular}

Using monofractal and GLCM analysis, it is shown that for six parameters of group IV neurons there was statistically significant difference between two parts of the human striatum, while seven parameters of group $\mathrm{V}$ neurons differed significantly. Precisely, in group IV, statistical significance was found in $D_{\text {out }}, D_{\text {skel }}, \Lambda, S_{A S M}, S_{I D M}$ and $S_{E}$. Consequently, group $\mathrm{V}$ cells between two parts of the striatum differs in $D_{\text {bin }}, \Lambda, S_{A S M}, S_{I D M}, S_{C O N}, S_{C O R}$ and $S_{E}$. More specifically, only one parameter of monofractal $(\Lambda)$ and three parameters of textural $\left(S_{A S M}, S_{I D M}\right.$ and $\left.S_{E}\right)$ analyses were significantly different between parts of the striatum in both groups of cells. Other parameters were different in a particular group only.

\section{Discussion}

\subsection{Aspiny Neurons}

In recent years much more attention has been focused on local circuit neurons, which, unequivocally, play an important role in striatal circuitry. They are phenotypically varied, distinct and precise in their properties, which allows them to modulate striatal function by taking part in the correct processing of corticostriatal information $[10,26]$. According to their morphology, neurochemistry and physiology, they are classified into the cholinergic interneurons and at least three groups of GABAergic interneurons $[9,10]$. The classification was made either by measuring diameter of the cell bodies or by qualitative estimation of the dendritic tree size. In our previous research we have measured five morphological properties of neurons by using eight morphometric parameters [11]. As we confirmed current qualitative classification, three groups of aspiny neurons (type III, IV and V) have been proposed.

In the present study, we used only two groups of striatal interneurons: group IV and group V. We spotted very obvious morphological differences between these two groups [11]. According to the dendritic field size, the distance of the dendritic branching points and terminations, type IV cells were classified as medium-sized aspiny neurons [9, 10]. However, the soma size, the shape of neurons, the dendritic aberrations and dendritic density clearly classified group $\mathrm{V}$ cells as small aspiny neurons, described in [10].

The shape of nerve cell reflects its role in communication [27] and in this sense results of the study can be interpreted. For instance, according to somatic size, it seems that the group IV neurons correspond to the cholinergic and the group $\mathrm{V}$ cells match up with parvalbumin-positive interneurons, observing their dendritic morphology [11]. As striatal projections are topographically organized (cognitive territory - most of the caudate nucleus and precommissural putamen; sensorimotor territory - dorsolateral rim of the head of caudate nucleus and postcommissural putamen; limbic territory - nucleus accumbens, olfactory tubercle and the most ventral parts of the caudate nucleus and putamen) we can assume that different morphology of the same neuronal type in different parts of the striatum can reflect functional diversity of the human striatum. These territories process cognitive, motor and emotional information, respectively. The motor loop is primarily associated with abnormal stereotypical motor behavior, the cognitive loop is presumably associated with inappropriate repetition of a goal, expressed in a relatively varied behavioral collection. The limbic loop is involved in motivational aspects of behavioral control, response to reward and obsessive-compulsive behavior [28].

Based on their electrophysiological properties, the activation of cholinergic neurons is usually slow and regular, their action potential lasts for a long time, and they are labeled as tonally active neurons (tonally active neuron, TAN) [29]. Cholinergic interneurons of the putamen are activated in response to motor signals from the cortex. The same cells of the caudate nucleus react to associative- 
cognitive information. Parvalvumin-positive GABAergic interneurons have been referred to as "fast-spiking, FS" interneurons [30]. They receive powerful excitatory impulses from the cortex, which differ from the impulses of the same origin that excite the projection cells [31]. Also, they receive cholinergic input information [32]. Target cells of parvalbumin-positive interneurons are medium-sized spiny neurons. Each of the GABAergic neostriatal interneuron monosynaptically causes inhibition of projection cells and creates strong inhibitory postsynaptic potential.

Cell body size, number of primary dendrites and their branching patterns reflect the ability of interneurons to make synaptic contacts. Morphological, as well as neurochemical and electrophysiological, heterogenity of the group IV and the group $\mathrm{V}$ human striatal neurons contribute in divergent nerve signal modulation and, consequently, in different responses. Since the caudate nucleus is mainly associative and putamen mainly sensorimotor part of the striatum, it could be expected that interneurons of the caudate nucleus, which process more complex neural information, have larger dendritic field area, as well as soma area than interneurons of the same group in the putamen.

\subsection{Computational Analysis}

Standard quantitative methods in science, i.e. their parameters, are based on classical Euclidean geometry [33]. Nevertheless, different studies from the end of the twentieth century showed that the most common biological patterns could be successfully characterized by fractal geometry [21]. The fractal analysis has been frequently used in addition to the other techniques [34]. Although there are no strict guidelines for the application of fractal analysis, the majority of researches tend to use box-counting method to calculate fractal dimension of digital images, making this method one of the most frequently used [22].

In this study we used parameters of standard fractal analysis, known as monofractal analysis [16]. Three parameters $\left(D_{\text {bin }}, D_{\text {out }}\right.$ and $\left.D_{\text {skel }}\right)$ quantified the space-filling property, shape and dendritic aberrations combined with the complexity of dendritic tree. We used them in our previous investigations $[11,35,36]$. For the first time, the neostriatal neurons were quantified by lacunarity, which assessed the homogeneity of the neuronal image in its simultaneous translation and rotation.

Since all neuronal images were saved in grayscale format before binarization, we were able to improve the results of the monofractal analysis. More precisely, we wanted to examine whether the texture parameters can bring new results in a quantitative analysis of the neuronal image. Like we stated before (Subsection 2.2), texture analysis aims to identify texture features of objects in an image by analyzing combinations of intensities at specified positions that are relative to each other in the image [37]. For that reason, it was enough to use only five from fourteen parameters, defined in [14].

We started the analysis by exploring the distribution of all parameters, so that we could see how to properly display them and continue to analyze them statistically. As we have previously pointed out (Section 3), there is no general way of checking distribution, but when the number of variables in the sample is greater than 100 , it is recommended to use the results of the Kolmogorov-Smirnov test [24]. As our results (Table 3) are not normally distributed, in further analyses we applied non-parametric statistical tests.

Then, we investigated whether there is a connection between two parameters of the monofractal [13] and the GLCM analysis [15]. We have shown that even though these parameters describe different image properties, there is a correlation between them (Figure 2). This has instructed us that in further analysis we should expect similar conclusions in these two parameters (Tables 4 and 5).

During the further analysis, we tested the difference between two groups of neurons in human striatum. The results showed that two neurons groups differ in three parameters of the monofractal and three parameters of the textural analysis. As noticed, between two groups of striatal neurons there were differences in space-filling ratio, shape of the neuron, dendritic aberrations and complexity of dendritic tree. When the texture of the picture was included in the analysis, we found that two neuronal groups differed in three parameters. The first parameter $\left(S_{A S M}\right)$ estimates the homogeneity of the image, while the other $\left(S_{C O N}\right)$ its heterogeneity. There was also a difference between the types in the gray-linear dependence of nearby pixels $\left(S_{C O R}\right)$.

Finally, between two parts of the neostriatum (putamen and caudate nucleus), morphological differences of both neuronal groups were examined. One parameter of the monofractal $(\Lambda)$ and four parameters of the GLCM $\left(S_{A S M}\right.$, $S_{I D M}, S_{C O N}$ and $S_{E}$ ) analysis differed in both neuronal groups (Table 5). The difference between neurons of the putamen and the caudate nucleus in other parameters was observed only in one neuronal group. More specifically, $D_{\text {out }}$ and $D_{\text {skel }}$ differed in group IV, while group $\mathrm{V}$ recorded a difference in $D_{\text {bin }}$ and $S_{C O R}$ (Table 5). The final results confirm the initial hypothesis (Section 1), since the morphological differences between neurons of two parts of the human neostriatum were noticed not only on binary but also in grayscale images.

\section{Conclusion}

The neostriatum is topographically organized in the sensorimotor, associative and limbic territories, and receives inputs from wide cortical areas. The morphological and morphometric study of the neostriatum neurons can provide valuable data about their connections and function. These facts reveal a pressing need to study the same neurons proceeding from different parts of the neostriatum.

A computational analysis of $2 \mathrm{D}$ neuronal images of the neostriatum performed in this study confirmed the existence of two groups of cells. Furthermore, our results confirmed previous morphological descriptions of local circuit neurons. Unlike previous studies, this report presents a complete monofractal analysis of neostriatal neurons, and for the first time investigates the grayscale images. The existence of 
differences between the two groups of local circuit neurons was confirmed by three (out of four) monofractal and three (out of five) GLCM features.

Although it is well known that the precommissural neostriatum receives input from cognitive cortical areas, it is considered that the putamen neurons belong to the sensorimotor striatal area while the caudate nucleus neurons belong to the cognitive area. Based on the significant morphometric differences found between the same type of nerve cells from different region of the striatum (putamen and caudate nucleus) we demonstrate the existence of morphometric differences of cells between the functionally identical striatal regions. In more detail, group IV differs between putamen and caudate nucleus by three parameters of monofractal and three parameters of GLCM analysis, while group $\mathrm{V}$ differs in only two monofractal and all five GLCM parameters.

These results confirmed our expectation that textural analysis has the advantage over other image analysis techniques, especially if the focus is on the morphological differences between the groups. But, the main conclusion of the present study is that texture analysis should be combined with other image analysis techniques. Another conclusion of this study is that research of neurons with the same morphological properties, but different topologies and functional diversity such as the spiny neurons from the neostriatum, should be continued.

\section{Acknowledgements}

This work was supported by Instituto Tecnológico de Santo Domingo (INTEC) of Dominican Republic (grant 0011389016-4) and Ministry of Education, Science and Technological Development of the Republic of Serbia (grant III41031).

\section{References}

[1] Pisani A, Centonze D, Bernardi G, Calabresi P, Striatal synaptic plasticity: implications for motor learning and Parkinson's disease. Mov Disord 20: 395-402, 2005.

[2] Krstonošic B, Morphological Analysis of Two Dimensional Projection of Neurons in the Human Neostriatum. Dissertation, Medical faculty, University of Novi Sad, Serbia, Balkans, 2013.

[3] Yin HH, Knowlton BJ, The role of the basal ganglia in habit formation. Nat Rev Neurosci 7: 464-476, 2006.

[4] Bernácer J, Prensa L, Giménez-Amaya JM, Chemical architecture of the posterior striatum in the human brain. $\mathrm{J}$ Neural Transm 115: 67-75, 2008.

[5] Steiner H, Tseng KY, Handbook of basal ganglia structure and function. Academic, London, 2010.

[6] Chang HT, Wilson CJ, Kitai ST, A Golgi study of rat neostriatal neurons: light microscopic analysis. J Comp Neurol 208: 107-126, 1982.
[7] Di Figlia M, Pasik T, Pasik P, Ultrastructure of Golgiimpregnated and gold-toned spiny and aspiny neurons in the monkey neostriatum. J Neurocytol 9: 471-492, 1980.

[8] Leontovich TA (1998) Large neostriatal neurons in humans and their possible role in neuronal networks. Neurosci Behav Physiol 28: 252-259.

[9] Braak H, Braak E, Neuronal types in the striatum of man. Cell Tissue Res 227: 319-342, 1982.

[10] Graveland GA, Williams RS, DiFiglia M, A Golgi study of the human neostriatum: neurons and afferent fibers. J Comp Neurol 234: 317-333, 1985

[11] Krstonošić B, Milošević NT, Gudović R, Marić DL, Ristanović D, Neuronal images of the putamen in the adult human neostriatum: revised classification supported with qualitative and quantitative analysis. Anat Sci Int 87: 115$125,2012$.

[12] Krstonošić B, Milošević NT, Marić DL, Babović SS, Quantitative analysis of spiny neurons in the adult human caudate nucleus: can it confirm the current qualitative ell classification? Acta Neurol Belg 115 (3): 273-280, 2015.

[13] Rajković N, Krstnošić B, Milošević N, Box-counting method of 2D neuronal image: method modification and quantitative analysis demonstrated on images from the monkey and human brain. Comp Math Methods Med https://doi.org/10.1155/2017/8967902,2017.

[14] Haralick R, Shanmugam K, Dinstein IH, Textural features for image classification. IEEE Trans Syst Man Cybern SMC-3: 610-621, 1973.

[15] Vujasinovic T, Pribic J, Kanjer K, Milošević NT, Tomasevic Z, Milovanovic Z, Nikolic-Vukosavljevic D, Radulovic M, Gray-level co-occurrence matrix texture analysis of breast tumor images in prognosis of distant metastasis risk. Microsc Microanal. 21 (3): 646-654, 2015.

[16] Rajkovic N, Kolarevic D, Kanjer K, Milosevic NT, NikolicVukosavljevic D, Radulovic M, Comparison of Monofractal, Multifractal and gray level Co-occurrence matrix algorithms in analysis of Breast tumor microscopic images for prognosis of distant metastasis risk. Biomed Microdevices https://doi.org/10.1007/s10544-016-0103-x,2016.

[17] Đuričić GJ, Radulović M, Sopta JP, Nikitović M, Milošević NT, Fractal and gray level coocurence matrix computational analysis of primary ostosarcoma magnetic resonance images: predicts the chemotherapy response. F Onc. https://doi.org/10.3389/fonc.2017.00246,2017.

[18] Cabrera J, Texture analyzer plugin. NIH, Image Processing and Analysis in Java, https://imagej.nih.gov/ij/plugins/texture.html,2006.

[19] Miloševic் NT, Fractal analysis of two dimensional images: parameters of the space-filling and shape, In Dumitrache I, Magda Florea A, Pop F, Dumitrascu A (Eds.) Proceedings of the 20th International Conference on Control Systems and Computer Science, Vol. 2: IAFA: Fractal Analysis of Medical Images, The Institute of Electrical and Electronics Engineers, Los Alamitos, pp 539-544, 2015.

[20] Smith Jr. TG, Lange GD, Marks WB, Fractal methods and results in cellular morphology - dimensions, lacunarity and multifractals. J Neurosci Meth 69 (2): 123-136, 1996. 
[21] Fernández E, and Jelinek HF, Use of fractal theory in neuroscience: methods, advantages, and potential problems. Methods 24 (4): 309-321, 2001

[22] Miloševic NT, The morphology of brain neurons: box counting method in quantitative analysis of 2D image. In: Di Ieva A (Ed). The Fractal Geometry of the Brain, SpringerVerlag, Berlin, Germany, pp 109-127, 2016.

[23] Karperien A, FracLac for ImageJ, version 2. 5. http://rsbinfonihgov/ij//fraclac/,2007.

[24] R. H. Riffenburgh RH, Statistics in Medicine, Academic Press, London, UK, 1999.

[25] Milošević NT, Krstonošić B, Elston GN, Rajković N, Boxcount analysis of two dimensional images: methodology, analysis and classification. In: Dumitrache I, Magda-Florea A, Pop F (eds.), Proceedings of $19^{\text {th }}$ International Conference on Control Systems and Computer Science, Vol. 2: Interdisciplinary approaches in fractal analysis, The Institute of Electrical and Electronics Engineers, Los Alamitos, CA, USA; pp 306-312, 2013.

[26] Lecumberri A, Lopez-Janeiro A, Corral-Domenge C, Berancer $\mathrm{J}$, Neuronal density and proportion of interneurons in the associative, sensorimotor and limbic human striatum. Brain Struct Funct 223 (4): 1615-1625, 2018.

[27] Ramón y Cajal S, Textura del sistema nervioso del hombre y de los vertebrados [Texture of the nervous system of man and the vertebrates]. Springer, New York, 1899.

[28] Haber S, Corticostriatal circuitry. Dialogues Clin Neurosci 18 (1): 7-21, 2016.

[29] Bennett BD, Wilson CJ, Spontaneous activity of neostriatal cholinergic interneurons in vitro. J Neurosci 19: 5586-5596, 1999.

[30] Kawaguchi Y, Physiological, morphological and histochemical characterization of three classes of interneurons in rat neostriatum. J Neurosci 13: 4908-4923, 1993.
[31] Ramanathan S, Hanley JJ, Deniau JM, Bolam JP, Synaptic convergence of motor and somatosensory cortical afferents onto GABAergic interneurons in the rat striatum. J Neurosci 22: 8158-8169, 2001.

[32] Chang HT, Kita H, Interneurons in the rat striatum: relationships between parvalbumin neurons and cholinergic neurons. Brain Res 574: 307-311, 1992.

[33] Zaletel I, Milošević NT, Todorović V, Kovačević-Filipović M, Puškaš N, Fractal and gray level co-occurrence matrix texture analysis of senescent and non-senescent deciduous teeth stem cells: a pilot study. Fractal Geometry and Nonlinear Anal in Med and Biol. 2 (2): 1-6, 2016.

[34] Milošević NT, Rajković N, Jelinek HF, Ristanović D, Richardson's method of segment counting versus boxcounting. In: Dumitrache I, Magda-Florea A, Pop F (eds.), Proceedings of $19^{\text {th }}$ International Conference on Control Systems and Computer Science, Vol. 2: Interdisciplinary approaches in fractal analysis, The Institute of Electrical and Electronics Engineers, Los Alamitos, CA, USA; pp 299-305, 2013.

[35] Milošević NT, Krstonošić B, Gudović R, Ristanović D, Fractal analysis of neuronal dendritic branching patterns in the human neostriatum: a revised classification scheme. In: Dobrescu R (Ed.), Proceedings CSCS-18, Vol. 2: Interdisciplinary approaches in fractal analysis, Editura Politehnica Press, Bucharest, Romania; pp 871-876, 2011.

[36] Jelinek HF, Milošević NT, Karperien A, Krstonošić B, BoxCounting and Multifractal Analysis in Neuronal and Glial Classification. In: Dumitrache I (Ed.), Advances In Intelligent Control Systems and Computers Science, Springer-Verlag, Berlin-Heidelberg, pp 177-190, 2013.

[37] Albregsten F, Statistical Texture Measures Computed from Gray Level Cooccurrence Matrices. Technical Note. Department of Informatics, University of Oslo, Norway, 1995. 